\title{
Tubazioni in polietilene per il trasporto di acqua
}



A. Pavan
R. Frassine

\section{Tubazioni}

in polietilene

per il trasporto

di acqua

Manuale per la progettazione,

la posa e la gestione

delle reti idriche

照 Springer 

A. PAVAN
R. FRASSINE

Politecnico di Milano, Milano

In allegato CD-ROM: Programma PiPEs

Springer-Verlag fa parte di Springer Science+Business Media

springer.it

(c) Springer-Verlag Italia, Milano 2005

ISBN 88-470-0268-0

Quest'opera è protetta dalla legge sul diritto d'autore. Tutti i diritti, in particolare quelli relativi alla traduzione, alla ristampa, all'uso di figure e tabelle, alla citazione orale, alla trasmissione radiofonica o televisiva, alla riproduzione su microfilm o in database, alla diversa riproduzione in qualsiasi altra forma (stampa o elettronica) rimangono riservati anche nel caso di utilizzo parziale. Una riproduzione di quest'opera, oppure di parte di questa, è anche nel caso specifico solo ammessa nei limiti stabiliti dalla legge sul diritto d'autore, ed è soggetta all'autorizzazione dell'Editore. La violazione delle norme comporta le sanzioni previste dalla legge.

L'utilizzo di denominazioni generiche, nomi commerciali, marchi registrati, ecc, in quest'opera, anche in assenza di particolare indicazione, non consente di considerare tali denominazioni o marchi liberamente utilizzabili da chiunque ai sensi della legge sul marchio.

Progetto e impaginazione: Valentina Greco, Milano

Progetto grafico della copertina: Simona Colombo, Milano

Stampa: Signum Srl, Bollate (MI) 


\section{Indice}

$\begin{array}{ll}\text { 1. Introduzione } & 1\end{array}$

2. II polietilene nel trasporto di acqua 5

2.1. La materia prima 5

$\begin{array}{ll}\text { 2.2. Proprietà meccaniche } & 7\end{array}$

2.3. Altre caratteristiche 14

3. Ciclo di produzione 19

3.1. Estrusione 20

3.2. Stampaggio ad iniezione 21

3.3. Controllo finale dei prodotti 23

3.4. Certificazione di prodotto 23

4. Progettazione della tubazione 25

4.1. Progettazione idraulica 25

4.2. Progettazione meccanica $\quad 35$

4.3. Resistenza meccanica alle onde sismiche 66

$\begin{array}{ll}\text { 4.4. } & \text { Opere fuori terra } \\ & 67\end{array}$

4.5. Ancoraggi 74

$\begin{array}{ll}\text { 4.6. Condotte subacquee } & 75\end{array}$

4.7. Curvature massime ammissibili 77

$\begin{array}{lll}\text { 4.8. Forza di trascinamento } & 78\end{array}$

4.9. Esempio di progettazione $\quad 80$

5. Giunzioni 87

$\begin{array}{lr}\text { 5.1. Saldature } & 87\end{array}$

5.2. Giunzioni per fissaggio meccanico 104

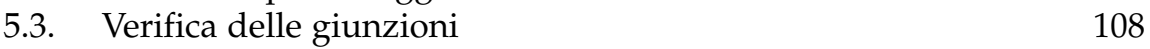

$\begin{array}{ll}\text { 5.4. Affidabilità delle reti } & 109\end{array}$

6. Tecniche di posa 111

6.1. Progettazione della trincea 111

6.2. Caratteristiche della trincea 114

6.3. Posa di condotte interrate 118

6.4. Tecnologie di posa trenchless 120

6.5. Realizzazione di attraversamenti e parallelismi 123

6.6. Posa in terreni contaminati 125

6.7. Effetto del cloro 125 
7. Capitolato d'opera e collaudo $\quad 127$

\begin{tabular}{ll} 
7.1. & Capitolato d'opera \\
\hline
\end{tabular}

$\begin{array}{ll}\text { 7.2. Collaudo } & 128\end{array}$

8. Esercizio, manutenzione, riparazione e riabilitazione delle reti 133

8.1. Perdite negli acquedotti 133

8.2. Sistemi di telecontrollo 136

8.3. Tecnologie per la riabilitazione 136

8.4. Tecnologie per la sostituzione 143

9. Vantaggi delle reti in polietilene 145

9.1. Vantaggi pratici 145

9.2. Vantaggi economici 148

9.3. Affidabilità e sicurezza 150

9.4. Mercato europeo del polietilene 151

9.5. Qualità dell'acqua 152

Appendice A. Struttura e proprietà del polietilene 153

A.1. Architettura delle molecole e cristallizzazione 153

A.2. Correlazioni proprietà-struttura 156

A.3. Viscoelasticità ed effetto della temperatura 157

A.4. Comportamento a lungo termine di tubi in pressione 160

A.5. Resistenza alla frattura per propagazione rapida 163

Appendice B. Disponibilità di tubi e raccordi 165

B.1. Tubi 165

$\begin{array}{lll}\text { B.2. Raccordi } & 167\end{array}$

Appendice C. La norma UNI EN 12201

C.1. Requisiti della materia prima $\quad 173$

$\begin{array}{ll}\text { C.2. Requisiti dei tubi } & 175\end{array}$

$\begin{array}{ll}\text { C.3. } & \text { Requisiti dei raccordi } \\ & 178\end{array}$

Appendice D. Norme e certificazioni $\quad 181$

D.1. Differenze tra decreto, circolare, norma e certificazione 181

D.2. Circolari e decreti 185

D.3. Normative 186

D.4. Qualificazione degli operatori 189

Tabelle

Elenco fonti delle figure $\quad 206$

$\begin{array}{ll}\text { Indice analitico } & 207\end{array}$ 


\section{Presentazione}

L'iniziativa di realizzare un manuale per la progettazione, la posa e la gestione di tubazioni in polietilene per il trasporto dell'acqua è nata dall'esigenza di raccogliere, elaborare e rendere disponibili ai progettisti delle reti idriche, al personale delle aziende di posa e ai tecnici di gestione dei servizi idrici le conoscenze sviluppate nel corso degli anni dai produttori di materia prima, dai trasformatori, dai laboratori di ricerca e dagli enti di normazione e certificazione.

Il manuale presenta dati, metodi e raccomandazioni utili per sviluppare appieno, nel rispetto delle normative vigenti sul territorio italiano, le potenzialità di sicurezza e affidabilità offerte dall'utilizzo del polietilene nel trasporto di acqua potabile. Esso vuol costituire uno strumento professionale di pratica utilità per la progettazione, realizzazione, installazione ed esercizio delle reti a partire dalla scelta dei materiali fino alla messa in opera o per la scelta delle tipologie di intervento per la riabilitazione di reti esistenti.

Gli argomenti trattati nel manuale sono parte di un patrimonio di conoscenze disponibile presso il Politecnico di Milano, e in particolare presso la Fondazione Laboratorio Prove Materie Plastiche (POLllampol). Tali conoscenze si sono sviluppate nel corso di un'attività pluridecennale di studio e caratterizzazione dei materiali e dei manufatti, di consulenza e di supporto tecnicoscientifico nel settore delle tubazioni in plastica, che ha permesso di attivare contatti molto stretti con l'intera filiera del settore e di esplorare a fondo le caratteristiche presentate dalle tubazioni in polietilene. Si è così evidenziato come il risultato finale dell'opera dipenda, oltre che da una sua corretta progettazione, che tenga conto della natura del materiale, anche dalle modalità di esecuzione che precedono la messa in servizio della tubazione.

Il contenuto del manuale è aggiornato allo stato dell'arte delle conoscenze e delle normative esistenti al momento della sua pubblicazione. Poiché non è possibile sviluppare appieno in un'opera di questa natura il dettaglio delle conoscenze disponibili e poiché le normative nazionali ed internazionali vengono continuamente modificate, si consiglia di approfondire e mantenere aggiornato il contenuto del manuale tramite il contatto costante con gli operatori e la consultazione continua delle normative e della letteratura tecnico-scientifica di settore.

Milano, ottobre 2004

Gli Autori

Gli autori desiderano ringraziare Giovanni Castiglioni, Patrizio Coldani, Angela Depinto, Gabriele Depinto e Davide Verzanini della Fondazione Laboratorio Prove Materie Plastiche (POLllampol) che hanno contribuito a vario titolo alla preparazione di questo manuale. Si ringrazia inoltre I'Ing. Francesco Briatico Vangosa, ricercatore del Politecnico di Milano, per lo sviluppo del programma di progettazione allegato. 